\title{
Prejuízo da vasodilatação muscular durante estresse mental em mulheres com hipotireoidismo subclínico
}

\author{
Impairment of muscle vasodilation during mental \\ stress in women with subclinical hypothyroidism
}

Fabiana de Faria Ghetti', Rafaela Pinheiro Lacerda', Francisco Zacaron

Wernek2 ${ }^{2}$ Emerson Filipino Coelho ${ }^{2}$, Mário Vaisman ${ }^{3}$, Jorge Roberto

Perrout de Lima', Daniel Godoy Martinez', Mateus Camaroti Laterza'

1 Unidade de Investigação

Cardiovascular e Fisiologia do

Exercício do Hospital Universitário e da Faculdade de Educação Física

e Desportos da Universidade

Federal de Juiz de Fora (UFJF)

Juiz de Fora, MG, Brasil

${ }^{2}$ Centro Desportivo da

Universidade Federal de Ouro Preto (Cedufop), Ouro Preto, MG, Brasil

${ }^{3}$ Serviço de Endocrinologia do Hospital Universitário Clementino Fraga Filho (HUCFF), Departamento de Clínica Médica, Faculdade de Medicina, Universidade

Federal do Rio de Janeiro (UFRJ), Rio de Janeiro, RJ, Brasil
Correspondência para: Mateus Camaroti Laterza Faculdade de Educação Física e Desportos,

Campus Universitário, Universidade Federal de Juiz de Fora 36330-160 - Juiz de Fora, MG, Brasil mateuslaterza@hotmail.com

Recebido em 28/Jan/2014 Aceito em 11/June/2014

DOI: 10.1590/0004-2730000003252

\section{RESUMO}

Objetivo: Testar a hipótese de que mulheres com hipotireoidismo subclínico (HSC) possuem condutância vascular do antebraço (CVA) prejudicados durante estresse mental. Sujeitos e métodos: Foram avaliadas 20 mulheres com HSC e 21 eutireoidianas (Grupo Controle), pareadas por idade $(p=0,699)$ e índice de massa corporal ( $p=0,462)$. O fluxo sanguíneo muscular (FSM), avaliado pela pletismografia de oclusão venosa, e a pressão arterial, medida pelo Dixtal2023, foram registrados simultaneamente durante 3 minutos de basal, seguidos de 3 minutos de estresse mental. A CVA foi calculada pela divisão do FSM pela pressão arterial média. Foi adotada significância de $p<0,05$. Resultados: 0 grupo HSC apresentou maior concentração do hormônio tireoestimulante $(7,57 \pm 3,17$ vs. $2,10 \pm 0,88 \mathrm{mU} / \mathrm{L}, \mathrm{p}<0,001)$. No basal, os grupos $\mathrm{HSC} \mathrm{e}$ Controle foram semelhantes respectivamente para FSM $(2,50 \pm 0,79$ vs. $2,55 \pm 0,71 \mathrm{~mL} / \mathrm{min} / 100$ $\mathrm{mL}, \mathrm{p}=0,905)$ e CVA $(2,80 \pm 0,90$ vs. 2,92 $\pm 0,88$ unidades, $\mathrm{p}=0,952)$. Durante todo o estresse mental, os grupos HSC e Controle aumentaram significativamente o FSM (efeito tempo, $\mathrm{p}<$ 0,001 ) e CVA (efeito tempo, $p<0,001$ ) em relação ao basal. Porém, essas variáveis foram significativamente menores no grupo HSC durante o primeiro (FSM: $3,66 \pm 0,96$ vs. 4,66 $\pm 1,61 \mathrm{~mL}$ / $\min / 100 \mathrm{~mL}, \mathrm{p}=0,018 ; \mathrm{CVA}: 3,95 \pm 1,08$ vs. $5,19 \pm 1,96$ unidades, $\mathrm{p}=0,010$ ) e segundo (FSM: 3,55 $\pm 1,01$ vs. $4,62 \pm 2,27 \mathrm{~mL} / \mathrm{min} / 100 \mathrm{~mL}, \mathrm{p}=0,018$; CVA: $3,75 \pm 1,07$ vs. $4,92 \pm 2,37$ unidades, $\mathrm{p}=$ $0,020)$ minutos do teste de estresse mental. Conclusão: Mulheres com HSC possuem comportamento vasodilatador prejudicado durante o estresse mental. Arq Bras Endocrinol Metab. 2014;58(7):750-7

Descritores

Hipotireoidismo; pressão arterial; vasodilatação

\section{ABSTRACT}

Objective: To test the hypothesis that women with subclinical hypothyroidism (SH) have forearm vascular conductance (FVC) impaired during mental stress. Subjects and methods: We evaluated 20 women with $\mathrm{SH}$ and 21 euthyroid (Control group), matched for age ( $\mathrm{p}$ $=0.699)$ and body mass index $(p=0.462)$. Muscle blood flow (MBF) was assessed by venous occlusion plethysmography and blood pressure by Dixtal2023. Both variables were recorded simultaneously for 3 minutes of baseline followed by 3 minutes of mental stress. The FVC was calculated by dividing MBF by mean arterial pressure. Significant differences were assumed at $\mathrm{p}<0.05$. Results: The $\mathrm{SH}$ group had higher concentrations of thyroid stimulating hormone $(7.57 \pm 3.17$ vs. $2.10 \pm 0,88 \mathrm{mU} / \mathrm{L}, \mathrm{p}<0.001)$. At baseline, the $\mathrm{SH}$ and control groups were similar for $\operatorname{MBF}(2.50 \pm 0.79$ vs. $2.55 \pm 0,71 \mathrm{~mL} / \mathrm{min} / 100 \mathrm{~mL}$, $\mathrm{p}=0.905$, respectively) and FVC $(2.80 \pm 0.90$ vs. $2.92 \pm 0.88$ units, $\mathrm{p}=0.952$, respectively $)$. Throughout the mental stress test the $\mathrm{SH}$ and Control groups increased the MBF (time effect, $p<0.001$ ) and FVC (time effect, $p<0.001$ ) compared to baseline protocol. However, these variables were lower in SH group during the first (MBF: $3.66 \pm 0.96$ vs. $4.66 \pm 1,61$ $\mathrm{mL} / \mathrm{min} / 100 \mathrm{~mL}, \mathrm{p}=0.018$, FVC: $3.95 \pm 1.08$ vs. $5.19 \pm 1,96$ units, $\mathrm{p}=0.010)$ and second (MBF: $3.55 \pm 1.01$ vs. $4.62 \pm 2,27 \mathrm{~mL} / \mathrm{min} / 100 \mathrm{ml}, \mathrm{p}=0.018 ; \mathrm{FVC}: 3.75 \pm 1.07$ vs. $4.92 \pm 2,37$ units, $\mathrm{p}=0.020$ ) minutes of mental stress test. Conclusion: Women with $\mathrm{SH}$ have reduced muscle vasodilatatory response during mental stress. Arq Bras Endocrinol Metab. 2014;58(7):750-7

\section{Keywords}

Hypothyroidism; blood pressure; vasodilation 


\section{INTRODUÇÃO}

$\mathrm{O}$ hipotireoidismo subclínico (HSC) é caracterizado por concentração sérica elevada do hormônio tireoestimulante (TSH) na presença de concentrações séricas normais de tiroxina livre (T4L) (1). A prevalência do HSC tem sido reportada entre 4 e $20 \%$ na população $(2,3)$, sendo maior no sexo feminino (4), em idosos (3), em caucasianos (2) e em regiões com ingestão excessiva de iodo (5). Apesar de na maioria dos casos não provocar sintomas, alterações mínimas na função da tireoide influenciam diretamente a fisiologia do sistema cardiovascular (6). De fato, em criteriosa metanálise foi observado que pacientes com HSC, com concentração sérica de TSH de 7,0 a 9,9 $\mathrm{mU} / \mathrm{L}$, apresentaram risco para desenvolvimento de eventos coronarianos de $17 \%$ e para morte por doenças cardiovasculares de $42 \%$ (7).

Uma das possíveis explicações para o risco aumentado de morbimortalidade de origem cardiovascular é a piora na função vascular (8). Em pacientes com insuficiência cardíaca, estágio final das doenças cardiovasculares, foi demonstrada associação inversa entre fluxo sanguíneo muscular (FSM) e mortalidade cardiovascu$\operatorname{lar}(9)$. Embora no HSC o FSM encontra-se preservado no repouso, a infusão de acetilcolina na artéria braquial não promoveu a esperada resposta vasodilatadora (10). Um dos possíveis mecanismos que justificam esse fenômeno é a menor biodisponibilidade do óxido nítrico, principalmente diante de situações que dependam da maior produção desse agente vasodilatador (10). Dessa forma, é possível sugerir que a maior probabilidade de eventos cardiovasculares nos pacientes com HSC esteja associada à piora da vasodilatação muscular, principalmente durante situações que solicitem maior ativação vascular.

A reação de fuga ou luta, evocada por situações agudas de estresse mental, é uma manobra fisiológica que estimula a vasodilatação muscular (11). Acredita-se que o prejuízo da vasodilatação muscular seja responsável, pelo menos em parte, pela hiper-reatividade pressórica durante o teste de estresse mental (12). Há claras evidências de que elevações agudas da pressão arterial promovem importantes lesões vasculares como, por exemplo, a ruptura da placa aterosclerótica, fato que resulta no evento isquêmico agudo do miocárdio e pior prognóstico desse paciente $(13,14)$. Porém, em pacientes com HSC não é conhecida a resposta vasodilatadora em situação de estresse mental. Dessa forma, o objetivo deste estudo foi testar a hipótese de que mulheres com HSC possuem, durante teste de estresse mental, FSM e condutância vascular do antebraço prejudicados, quando comparadas às eutireoidianas.

\section{SUJEITOS E MÉTODOS}

\section{Amostra}

Pelo cálculo amostral baseado em artigo publicado anteriormente (10), utilizando poder de $90 \%$ e nível de significância de $5 \%$, foi constatado que deveriam ser recrutadas no mínimo 13 voluntárias em cada grupo. Foram avaliadas 20 mulheres com diagnóstico de HSC (Grupo HSC) e, adicionalmente, como controle, 21 mulheres eutireoidianas (Grupo Controle), recrutadas dos Serviços de Endocrinologia do Hospital e Maternidade Terezinha de Jesus, Juiz de Fora, Minas Gerais. Os grupos foram pareados por idade e índice de massa corporal (IMC) e todas as voluntárias tinham idade entre 18 e 59 anos. Além disso, no grupo HSC foram incluídas mulheres com diagnóstico prévio da disfunção tireoidiana, enquanto no grupo Controle foram incluídas mulheres com TSH e T4L dentro da faixa de referência para o kit utilizado, e anticorpos antitireoperoxidase (aTPO) negativo. Para ambos os grupos, os critérios de não inclusão foram: uso de levotiroxina e/ou de drogas que pudessem interferir na função tireoidiana, presença de doenças, mesmo que estáveis, que pudessem interferir na concentração circulante dos hormônios tireoidianos; gestação, tabagismo e/ou prática de exercício físico regular nos últimos seis meses.

Todas as voluntárias, somente após esclarecimento prévio e concordância, assinaram o Termo de Consentimento Livre e Esclarecido. Este estudo foi aprovado pelo Comitê de Ética e Pesquisa com Seres Humanos do Hospital Universitário da Universidade Federal de Juiz de Fora (Parecer n 374/2011), Minas Gerais.

\section{Avaliação antropométrica}

O peso corporal foi avaliado por meio da balança da marca Filizola, com precisão de $0,1 \mathrm{~kg}$. A estatura foi obtida por meio do estadiômetro acoplado à balança Filizola, com precisão de $0,5 \mathrm{~cm}$. O IMC foi calculado a partir da relação do peso pela estatura ao quadrado. Para as medidas de circunferência da cintura e do pescoço, foi utilizada fita métrica da marca Hokanson ${ }^{\circledR}$, com precisão de $0,1 \mathrm{~cm}$. As medidas antropométricas foram feitas seguindo a padronização da Sociedade Internacional para o Progresso da Cineantropometria 
(International Society for Advancement in Kinanthropometry - ISAK) (15).

\section{Avaliação laboratorial}

O TSH, T4L e aTPO foram determinados por meio de ensaio imunométrico por quimioluminescência de terceira geração, utilizando o aparelho automático Access $2^{\circledR} \mathrm{e}$ kit Beckman Coulter ${ }^{\circledR}$. O HSC foi definido por concentração de TSH maior que $4,94 \mathrm{mU} / \mathrm{L}$ e nível de T4L entre 0,70 e 1,48 ng/dL, nas duas medidas realizadas em dias distintos, com intervalo mínimo de quatro semanas entre esses dias. Os níveis séricos de colesterol total, HDL-c, LDL-c, triglicérides e glicemia de jejum foram determinados por meio de ensaio enzimático colorimétrico.

\section{Pressão arterial e frequência cardíaca}

Para aferição da pressão arterial e da frequência cardíaca (FC), a voluntária foi posicionada em decúbito dorsal. A pressão arterial sistólica (PAS), a pressão arterial diastólica (PAD) e pressão arterial média (PAM) foram aferidas automaticamente, de forma não invasiva, no membro inferior direito, por meio do monitor multiparamétrico DIXTAL, modelo 2023. A FC foi registrada continuamente, por meio de cinco eletrodos cutâneos, posicionados de acordo com a derivação padrão fornecida pelo mesmo monitor multiparamétrico (16).

\section{Fluxo sanguíneo muscular}

O FSM do antebraço foi avaliado por meio do método não invasivo de pletismografia de oclusão venosa da marca Hokanson ${ }^{\circledR}$ (16). As voluntárias foram posicionadas em posição dorsal com o braço não dominante elevado no nível acima do coração, para adequada drenagem venosa. Um tubo silástico preenchido com mercúrio, conectado a um transdutor de baixa pressão, foi colocado ao redor do antebraço a cinco centímetros de distância da articulação úmero-radial. Um manguito foi colocado no punho e insuflado a nível suprassistólico $(200 \mathrm{mmHg})$, um minuto antes de iniciar as medidas, e assim permaneceu durante todo o protocolo experimental, com objetivo de bloquear o fluxo sanguíneo para a mão. Outro manguito foi colocado no braço e insuflado, em intervalos de 15 segundos, à pressão de $60 \mathrm{mmHg}$, durante 7 a 8 segundos, e em seguida desinflado rapidamente e mantido pelo mesmo tempo. Esse procedimento totalizou 4 ciclos por minuto. O aumento da tensão no tubo silástico refletiu o aumento do volume do antebraço e, consequentemente, o fluxo sanguíneo nesse seguimento, determinado $\mathrm{em} \mathrm{mL} / \mathrm{min} / 100 \mathrm{~mL}$ de tecido. A aquisição do sinal da onda do FSM foi obtida em tempo real em um computador por meio do programa Non Invasive Vascular Program 3. A avaliação da condutância vascular do antebraço (CVA) foi realizada pela divisão do FSM periférico pela PAM $(\mathrm{mmHg})$, multiplicada por 100 e expressa em "unidades" (16).

\section{Teste de estresse mental}

O protocolo de estresse mental foi conduzido pelo mesmo avaliador por meio do Teste de Conflito de Cores - Stroop Color Word Conflict Test (17). Este teste consiste em uma série de palavras de cores pintadas em cores incongruentes. A FC, pressão arterial e FSM foram registrados simultaneamente durante 3 minutos basais seguidos de 3 minutos de estresse mental. As voluntárias foram orientadas a pronunciar a cor da tinta com que a palavra foi pintada e não ler a palavra, sendo advertida na ocorrência de erro. Ao final do teste foi questionado à voluntária como foi a experiência do teste, categorizando a intensidade do estresse percebido pela seguinte escala: 0 = não estressante; 1 = pouco estressante; 2 = estressante; 3 = muito estressante; $4=$ extremamente estressante (12).

\section{Protocolo experimental}

A pesquisa foi realizada ao longo de três dias não consecutivos, separados por no mínimo 48 horas. No primeiro dia, foi realizada a avaliação antropométrica. No segundo dia, a voluntária compareceu ao Laboratório, após jejum de 12 horas, e foi submetida à coleta de sangue. No terceiro dia, a voluntária respondeu aos questionários de ansiedade e depressão e foi submetida à avaliação simultânea da PAS, PAD, PAM, FC e FSM por 3 minutos, caracterizando o período Repouso. $\mathrm{E}$ mais todas essas variáveis hemodinâmicas foram registradas simultaneamente durante o teste de estresse mental, composto pelos períodos Basal e Teste. Para a realização desse protocolo, a voluntária foi orientada, previamente, a ingerir refeição leve até uma hora antes do início da avaliação do fluxo sanguíneo, a não ingerir bebidas com cafeína ou bebidas alcoólicas e a não praticar atividade física vigorosa nas 24 horas anteriores aos experimentos. Todos os procedimentos foram realizados no período da manhã. 


\section{Análise estatística}

Os dados são descritos como média \pm desvio-padrão. O teste de Shapiro-Wilk foi utilizado para testar o pressuposto de normalidade das variáveis. As características clínicas, bioquímicas e hemodinâmicas no repouso dos grupos foram comparadas usando o teste $t$ de Student para amostras independentes, o teste U de Mann-Whitney e Qui-quadrado. A análise de variância $2 \times 4 \mathrm{com}$ medida repetida no segundo fator, seguida do post hoc LSD de Fisher, foi utilizada para testar as possíveis diferenças no comportamento hemodinâmico entre os grupos, no basal e ao longo do teste de estresse mental. O tamanho do efeito das diferenças significativas entre os grupos foi calculado pelo $d$ de Cohen. Adotou-se a seguinte classificação para o tamanho do efeito: 0,20 $\leq d<50=$ pequeno; $0,50 \leq d<0,80=$ moderado; $d$ $\geq 0,80=$ elevado. A significância estatística foi estabelecida quando o $\mathrm{p}<0,05$. Todas as análises estatísticas foram realizadas por meio do programa STATISTICA (versão 5; StatSoft, USA).

\section{RESULTADOS}

Os grupos foram semelhantes em relação ao IMC, circunferência da cintura e do pescoço, estado menopausal, T4L, perfil lipídico e hemodinâmica no repouso (Tabela 1). Como esperado, o grupo HSC apresentou concentração significativamente maior de TSH (Tabela 1 ) e $38 \%$ apresentaram aTPO positivo. Além disso, a glicemia de jejum foi significativamente maior no grupo HSC (Tabela 1).

Durante o teste de estresse mental, os valores de PAD, PAM e FC aumentaram de modo similar (efeito interação; $\mathrm{p}=0,713, \mathrm{p}=0,304$ e $\mathrm{p}=0,231$, respectivamente) e significativo no primeiro (efeito tempo; $\mathrm{p}=0,02, \mathrm{p}<0,001$ e $\mathrm{p}<0,001$, respectivamente $),$ segundo (efeito tempo; $\mathrm{p}<0,001, \mathrm{p}<0,001$ e $\mathrm{p}<$ 0,001 , respectivamente) e terceiro (efeito tempo; $\mathrm{p}<$ $0,001, \mathrm{p}<0,001$ e p $<0,001$, respectivamente) minutos em relação ao basal, nos grupos HSC e Controle (Tabela 2). E para todas essas variáveis não foram identificadas diferenças significativas entre os grupos (efeito grupo; $\mathrm{p}=0,527, \mathrm{p}=0,664$ e $\mathrm{p}=0,126$, respectivamente) (Tabela 2). Em relação ao basal, os valores de PAS aumentaram significativamente durante o segundo (efeito tempo; $\mathrm{p}<0,001$ ) e terceiro (efeito tempo; $\mathrm{p}$ $<0,001)$ minutos do estresse mental, nos grupos HSC e Controle. Durante todo o teste de estresse mental, o comportamento da PAS foi similar entre os grupos (efeito interação; $p=0,261$ ) e não foi verificada diferença significativa nos níveis pressóricos (efeito grupo; $\mathrm{p}=0,851$ ) (Tabela 2).

Tabela 1. Características clínicas, laboratoriais e hemodinâmicas no repouso de mulheres com hipotireoidismo subclínico (Grupo HSC) e eutireoidianas (Grupo Controle)

\begin{tabular}{lccc}
\hline Variáveis & $\begin{array}{c}\text { Grupo } \\
\text { HSC } \\
\text { (n= 20) }\end{array}$ & $\begin{array}{c}\text { Grupo } \\
\text { Controle } \\
(\mathbf{n}=\mathbf{2 1})\end{array}$ & p-valor \\
\hline Idade (anos) & $37 \pm 11$ & $38 \pm 10$ & 0,699 \\
IMC (kg/m²) & $26 \pm 5$ & $25 \pm 5$ & 0,462 \\
Cintura (cm) & $81 \pm 11$ & $78 \pm 10$ & 0,424 \\
Pescoço (cm) & $34 \pm 2$ & $33 \pm 2$ & 0,475 \\
Menopausa (n/\%) & $3 / 15$ & $4 / 20$ & 0,731 \\
TSH (mU/L)* & $7,57 \pm 3,17$ & $2,10 \pm 0,88$ & $<0,001$ \\
T4L (ng/dL) & $0,99 \pm 0,15$ & $1,04 \pm 0,11$ & 0,236 \\
CT (mg/dL) & $210 \pm 44$ & $203 \pm 39$ & 0,565 \\
LDL (mg/dL) & $134 \pm 35$ & $127 \pm 35$ & 0,536 \\
HDL (mg/dL) & $51 \pm 11$ & $53 \pm 13$ & 0,504 \\
Triglicérides (mg/dL) & $131 \pm 77$ & $112 \pm 62$ & 0,383 \\
Glicose (mg/dL)** & $90 \pm 9$ & $82 \pm 8$ & 0,010 \\
PAS (mmHg) & $125 \pm 8$ & $123 \pm 13$ & 0,625 \\
PAD (mmHg) & $66 \pm 6$ & $66 \pm 8$ & 0,853 \\
PAM (mmHg) & $86 \pm 6$ & $85 \pm 9$ & 0,788 \\
FC (bpm) & $70 \pm 9$ & $69 \pm 11$ & 0,861 \\
FSM (ml/min/100ml) & $2,28 \pm 0,67$ & $2,50 \pm 0,98$ & 0,441 \\
CVA (unidades) & $2,63 \pm 0,80$ & $2,90 \pm 1,07$ & 0,396 \\
\hline
\end{tabular}

IMC: índice de massa corporal; TSH: hormônio estimulante da tireoide; T4L: tiroxina livre; CT: colesterol total; LDL: lipoproteína de baixa densidade; HDL: lipoproteína de alta densidade; PAS: pressão arterial sistólica; PAD: pressão arterial diastólica; PAM: pressão arterial média; FC: frequência cardíaca; FSM: fluxo sanguíneo muscular; CVA: condutância vascular do antebraço. ${ }^{*} p<0,05$, teste Mann-Whitney U; ${ }^{\star \star} p<0,05$, teste " $t$ " Student.

Tabela 2. Pressão arterial e frequência cardíaca durante 0 estresse mental

\begin{tabular}{|c|c|c|c|c|c|}
\hline & & \multicolumn{4}{|c|}{ Estresse mental } \\
\hline & & Basal & $1^{0} \mathrm{~min}$ & $2^{0} \min$ & $3^{\circ} \mathrm{min}$ \\
\hline \multirow{3}{*}{$\begin{array}{l}\text { PAS } \\
\text { (mmHg) }\end{array}$} & Grupo HSC & $130 \pm 11$ & $145 \pm 16$ & $150 \pm 15^{\star}$ & $151 \pm 17^{\star}$ \\
\hline & $\begin{array}{l}\text { Grupo } \\
\text { Controle }\end{array}$ & $128 \pm 10$ & $135 \pm 12$ & $141 \pm 12^{*}$ & $138 \pm 13^{*}$ \\
\hline & Grupo HSC & $70 \pm 7$ & $77 \pm 12^{*}$ & $81 \pm 12^{*}$ & $80 \pm 10^{*}$ \\
\hline \multirow[t]{2}{*}{$\begin{array}{l}\text { PAD } \\
\text { (mmHg) }\end{array}$} & $\begin{array}{l}\text { Grupo } \\
\text { Controle }\end{array}$ & $68 \pm 6$ & $71 \pm 7^{*}$ & $73 \pm 8^{*}$ & $72 \pm 8^{*}$ \\
\hline & Grupo HSC & $90 \pm 8$ & $100 \pm 13^{*}$ & $104 \pm 13^{*}$ & $104 \pm 11^{*}$ \\
\hline \multirow[t]{2}{*}{$\begin{array}{l}\text { PAM } \\
(\mathrm{mmHg})\end{array}$} & $\begin{array}{l}\text { Grupo } \\
\text { Controle }\end{array}$ & $88 \pm 7$ & $92 \pm 8^{\star}$ & $96 \pm 7^{*}$ & $94 \pm 9^{*}$ \\
\hline & Grupo HSC & $67 \pm 8$ & $77 \pm 15^{\star}$ & $75 \pm 12^{*}$ & $74 \pm 14^{*}$ \\
\hline $\begin{array}{l}\mathrm{FC} \\
\text { (bpm) }\end{array}$ & $\begin{array}{l}\text { Grupo } \\
\text { Controle }\end{array}$ & $67 \pm 10$ & $90 \pm 19^{*}$ & $85 \pm 16^{*}$ & $85 \pm 15^{*}$ \\
\hline
\end{tabular}

Valores: média \pm desvio-padrão. PAS: pressão arterial sistólica; PAD: pressão arterial diastólica; PAM: pressão arterial média; FC: frequência cardíaca. * Diferença significativa em relação ao basal $(\mathrm{p}<0,001 ;$ ANOVA). 
Apesar de serem semelhantes no basal $(\mathrm{p}=0,905 \mathrm{e}$ $\mathrm{p}=0,952$, respectivamente), durante o estresse mental, o comportamento do FSM e o da CVA foram significativamente diferentes entre os grupos HSC e Controle (efeito interação; $\mathrm{p}<0,010$ e $\mathrm{p}<0,001$, respectivamente; Figura 1). Tanto o grupo HSC quanto o grupo Controle aumentaram significativamente o FSM (efeito tempo, $\mathrm{p}<0,001$ ) e CVA (efeito tempo, $\mathrm{p}<0,001$ ) em relação ao basal. Porém, essas variáveis foram significativamente menores no grupo HSC durante o primeiro (FSM: $\mathrm{p}=0,018$; CVA: $\mathrm{p}=0,010$ ) e segundo (FSM: $\mathrm{p}=0,018 ; \mathrm{CVA}: \mathrm{p}=0,020)$ minutos do teste de estresse mental. O tamanho do efeito entre os grupos no FSM e na CVA foi, respectivamente, de 0,78 (moderado) e 0,81 (elevado) no primeiro minuto, e de 0,65 (moderado) e 0,68 (moderado) no segundo minuto do estresse.

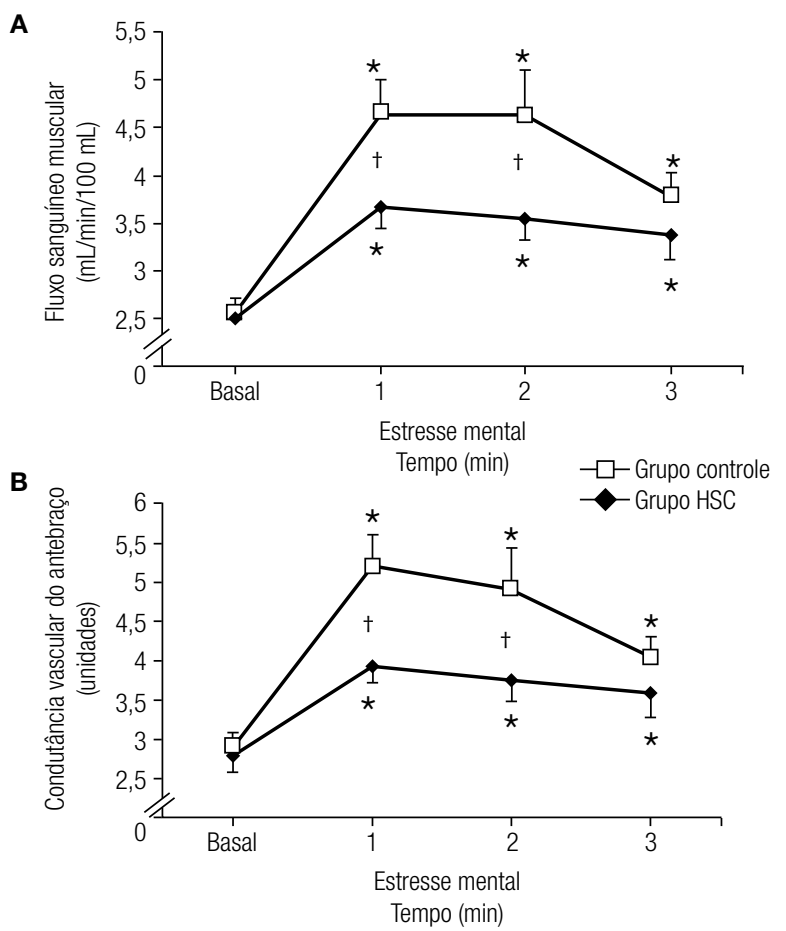

Figura 1. Fluxo sanguíneo muscular do antebraço (A) e condutância vascular do antebraço $(\mathbf{B})$ durante o estresse mental. * Diferença significativa em relação ao basal $(p<0,001)$; $†$ diferença significativa entre os grupos $(p<0,05)$.

Após a conclusão do teste de conflito de cores, não houve diferenças significativas em relação aos níveis de estresse percebido entre os grupos HSC e Controle $($ Mediana $=1$ vs. 1 , respectivamente; $\mathrm{p}=0,393)$.

\section{DISCUSSÃO}

O principal achado desse estudo foi que mulheres com HSC, relativamente jovens e sem história de condições que pudessem interferir no risco cardiovascular, apresentaram prejuízo do FSM e da CVA durante uma manobra fisiológica de estresse mental, quando comparadas a mulheres eutireoidianas. O tamanho do efeito da diferença na CVA entre os grupos, durante o primeiro minuto do estresse mental, indica que esse achado é bastante relevante em termos de significância prática e parece ser resultante da disfunção tireoidiana subclíni$\mathrm{ca}$, considerando que os grupos HSC e Controle foram similares quanto a possíveis variáveis intervenientes dessa relação, como idade, IMC, circunferência da cintura e do pescoço, perfil lipídico e nível de intensidade de estresse percebido.

Poucos estudos $(10,18-20)$ avaliaram a reatividade vascular no HSC. Avaliamos o comportamento do FSM por meio do método não invasivo de pletismografia de oclusão venosa, considerada "padrão-ouro" para o estudo da fisiologia vascular humana in vivo (21). Taddei e cols. (10) foram pioneiros em utilizar esse método para avaliar o fluxo sanguíneo de indivíduos com HSC, com infusão de acetilcolina na artéria braquial. Mas a primeira evidência científica a descrever a relação negativa entre o HSC e a vasodilatação foi publicada em 1997 por Lekakis e cols. (22), utilizando o método da medida da dilatação fluxo-mediada da artéria braquial.

Os resultados encontrados por nosso estudo e por Taddei e cols. (10) sustentam a evidência inicial (22) de que a função vascular no HSC, durante manobras fisiológicas, encontra-se prejudicada. De fato, Taddei e cols. (10) demonstraram que a resposta de fluxo sanguíneo à infusão de acetilcolina intrabraquial, um vasodilatador dependente do endotélio, encontrou-se significativamente diminuída em catorze pacientes (11 mulheres) hipercolesterolêmicos, com HSC, em relação a catorze controles normocolesterolêmicos e a catorze controles também dislipidêmicos. Além do desfecho, algumas características dos pacientes com HSC foram bem semelhantes entre nossos estudos, como idade, IMC, concentração sérica de TSH e colesterol total.

O prejuízo da função endotelial no HSC também foi demonstrado por Kilic e cols. (18). Nesse estudo, após a manobra fisiológica de hiperemia reativa, a dilatação fluxo-mediada era significativamente menor nos pacientes com HSC quando comparados aos controles, enquanto a vasodilatação independente do endotélio, provocada por nitroglicerina sublingual, era similar entre eles (18). Entretanto, Türemen e cols. (19) observaram que tanto a dilatação dependente do endotélio quanto a resposta à nitroglicerina estavam prejudicadas 
nos indivíduos com HSC. Em nosso estudo, as respostas prejudicadas de FSM e a CVA podem estar relacionadas a fatores dependentes e/ou independentes do endotélio.

Ao contrário das evidências suportadas anteriormente, Cabral e cols. (20) não encontraram associação significativa entre função tireoidiana e parâmetros vasculares. Nessa investigação, foram comparadas $21 \mathrm{mu}$ lheres com HSC $(42,4 \pm 10,8$ anos) e 21 eutireoidianas $(44,2 \pm 8,5$ anos $)$. Apesar de o TSH das pacientes com HSC $(8,3 \pm 4,3 \mathrm{mIU} / \mathrm{dL})$ ser, em média, maior que o valor observado no presente estudo, a dilatação fluxo-mediada foi semelhante entre casos e controles. E, mesmo com a subdivisão do grupo HSC em baixo $(\geq 4 \mathrm{e}>8 \mathrm{mU} / \mathrm{L})$ ou alto $(\geq 8 \mathrm{e}<12 \mathrm{mU} / \mathrm{L})$ nível de TSH, nenhuma diferença foi observada na dilatação fluxo-mediada (20).

Ainda, para minimizar possível limitação na interpretação dos resultados, corrigimos o FSM pela pressão de perfusão, obtendo a CVA. E, mesmo assim, a piora na função vascular se manteve no grupo de pacientes com a disfunção tireoidiana subclínica. Dessa forma, esse é o primeiro estudo a mostrar que mulheres com HSC apresentam comportamento da condutância vascular prejudicado diante de uma situação estressora.

Uma das possíveis explicações para o prejuízo da resposta vascular em mulheres com HSC, durante o estresse mental, seria a redução na disponibilidade de óxido nítrico. Taddei e cols. (23), ao medirem nos indivíduos com HSC o fluxo sanguíneo no antebraço durante infusão de acetilcolina, observaram que a resposta de vasodilatação dependente do endotélio é resistente ao L-NMMA, um inibidor do óxido nítrico. Segundo os autores, a disfunção endotelial presente nesses pacientes com tireoidite de Hashimoto foi causada, em parte, pela inflamação sistêmica subclínica, que promoveu aumento do estresse oxidativo (23).

A dislipidemia é outro fator que pode contribuir para a disfunção endotelial no HSC (23). A hipercolesterolemia diminui a expressão da óxido nítrico sintase endotelial e aumenta os níveis de dimetilarginina assimétrica, um marcador do estresse oxidativo (24). Porém, no presente estudo não encontramos diferença no LDL colesterol entre o grupo HSC e grupo Controle (Tabela 1).

A hiperglicemia, em estados de resistência à insulina, também tem papel importante na disfunção endotelial. Provavelmente, níveis elevados de glicemia aumentam o estresse oxidativo (25), as citocinas inflamatórias (26) e reduzem a síntese de óxido nítrico em associação com elevados níveis de dimetilarginina assimétrica (25). Em nosso estudo, não avaliamos a resistência à insulina, porém identificamos que a glicemia de jejum era significativamente mais elevada nas mulheres do grupo HSC que nas eutireoidianas, embora os valores de glicemia de ambos os grupos estivessem dentro dos valores de normalidade.

Além desses possíveis mecanismos, o TSH poderia explicar a diminuição do FSM e da CVA das pacientes com HSC. Dardano e cols. (27) demonstraram que o aumento agudo dos níveis séricos de TSH, por meio da administração de TSH recombinante em pacientes com carcinoma de tireoide, prejudicou a vasodilatação dependente do endotélio e aumentou os níveis circulantes de IL-6, TNF- $\alpha$ e lipoperoxidase. Esses achados sugerem que o TSH parece exercer efeito sistêmico, incluindo a produção de citocinas, que alteram a disponibilidade do óxido nítrico, levando à disfunção do endotélio vascular pelo aumento do estresse oxidativo (20).

A piora na função vascular é uma das possíveis explicações para o risco aumentado de morbimortalidade de origem cardiovascular (8). Avaliando o fluxo sanguíneo do antebraço pela mesma metodologia aplicada no presente estudo, Munhoz e cols. (9) demonstraram que o baixo FSM no repouso foi determinante na maior probabilidade de morte de origem cardiovascular em pacientes com insuficiência cardíaca, após um ano de seguimento. Embora as pacientes com HSC apresentassem no repouso FSM e CVA semelhante às eutireoidianas, o sistema cardiovascular não apresentou a esperada resposta vasodilatadora durante uma situação de estresse mental. Foi mostrado que os ajustes hemodinâmicos ocorridos durante estresse mental, induzido em laboratório, são semelhantes às situações estressantes do cotidiano (28). Dessa forma, podemos sugerir que, em situações agudas de estresse, o prejuízo no FSM e na CVA poderia ser responsável, pelo menos em parte, pela maior probabilidade de eventos cardiovasculares em mulheres com HSC. De fato, em criteriosa metanálise, baseada em dados individuais de 11 estudos prospectivos, o HSC foi associado significativamente ao risco de desenvolvimento e morte por doença arterial coronariana (7). Além disso, neste estudo foi observado que os riscos desses desfechos foram superiores para nível de TSH maior ou igual a $10 \mathrm{mU} / \mathrm{L}$, porém indivíduos com TSH entre 7,0 e 9,9 mU/L já apresentam $17 \%$ de risco para eventos coronarianos e $42 \%$ de risco para morte por doenças cardiovasculares (7). 
Em relação às medidas de frequência cardíaca e de pressão arterial, não encontramos diferenças significativas entre os grupos HSC e controles, no repouso e durante o teste de estresse mental. Além disso, os grupos apresentaram a resposta natural de aumento dessas variáveis hemodinâmicas ao longo do teste. No repouso, estudos observaram que a medida clínica da pressão arterial é semelhante entre pacientes com HSC e eutireoidianos $(10,18)$. No entanto, por meio da monitorização ambulatorial da pressão arterial, Ferreira e cols. (29) encontraram correlação positiva entre os níveis de PAD e valores séricos de TSH em pacientes com HSC $(\mathrm{r}=0,477 ; \mathrm{p}=, 004)$. Por essa razão, esses autores sugerem que a progressão de HSC para níveis mais elevados de TSH pode aumentar o risco cardiovascular pelo aumento da PAD (29).

O presente estudo é limitado por não ter avaliado os níveis de cortisol, reconhecido como o principal hormônio do estresse mental (30). Esse dado nos permitiria quantificar o nível de estresse induzido às voluntárias. Entretanto, ao final do teste, a intensidade do estresse percebido foi semelhante entre os grupos HSC e Controle. E, por não haver diferença significativa, confiamos que o estímulo estressor foi semelhante entre os grupos estudados. Portanto, os resultados obtidos nos permitem concluir que mulheres com HSC possuem FSM e condutância vascular do antebraço prejudicados durante o estresse mental.

Declaração: os autores declaram não haver conflitos de interesse científico neste estudo.

\section{REFERÊNCIAS}

1. Weiss IA, Bloomgarden N, Frishman WH. Subclinical hypothyroidism and cardiovascular risk: recommendations for treatment. Cardiol Review. 2011;19(6):291-9.

2. Hollowell JG, Staehling NW, Flanders WD, Hannon WH, Gunter EW, Spencer CA, et al. Serum TSH, T4, and thyroid antibodies in the United States Population (1988 to 1994): National Health and Nutrition Examination Survey (NHANES III). J Clin Endocrinol Metab. 2002;87:489-99.

3. Canaris GJ, Manowitz NR, Mayor G, Ridgway C. The Colorado thyroid disease prevalence study. Arch Intern Med. 2000;160:52634.

4. Cappola AR, Fried LP, Arnold AM, Danese MD, Kuller LH, Burke GL, et al. Thyroid status, cardiovascular risk, and mortality in older adults: The Cardiovascular Health Study. JAMA. 2006;295(9):1033-41.

5. Lauberg P, Pedersen KM, Hreidarsson A, Sigfusson N, Iversen E, Knudsen PR. lodine intake and the pattern of thyroid disorders: a comparative epidemiological study of thyroid abnormalities in the elderly in Iceland and in Jutland, Denmark. J Clin Endocrinol Metab. 1998;83:765-9.
6. Rodondi N, Newman AB, Vittinghoff E, Rekeneire N, Satterfield S, Harris TB, et al. Subclinical hypothyroidism and the risk of heart failure, other cardiovascular events, and death. Arch Intern Med. 2005;165:2460-6.

7. Rodondi N, Den Elzen WPJ, Bauer DC, Cappola AR, Razvi S, Walsh $\mathrm{JP}$, et al. Subclinical hypothyroidism and the risk of coronary heart disease and mortality. JAMA. 2010;304(12):1365-74.

8. Bacon SL, Lavoie KL, Arsenault A, Dupuis J, Pilote L, Laurin L. et al. The research on endothelial function in women and men at risk for cardiovascular disease (REWARD) study: Methodology. BMC. 2011;11:50.

9. Munhoz RT, Negrão CE, Barretto ACP, Ochiai ME, Cardoso JNC, Morgado PC, et al. Microneurografia e pletismografia de oclusão venosa na insuficiência cardíaca: correlação com prognóstico. Arq Bras Cardiol. 2009;92(1):46-53.

10. Taddei S, Caraccio N, Virdis A, Dardano A, Versari D, Ghiadoni L, et al. Impaired endothelium-dependent vasodilatation in subclinical hypothyroidism: beneficial effect of levothyroxine therapy. $J$ Clin Endocrinol Metab. 2003;88(8):3731-7.

11. Dietz N, Rivera JM, Eggener SE, Warner RTFO, Joyner MJ. Nitric oxide contributes to the rise in forearm blood flow during mental stress in humans. J Physiol. 1994;480(2):361-8.

12. Fernandes PROF, Lira FAS, Borba VVL, Costa MJC, Trombeta IC, Santos MSC, et al. Vitamin $C$ restores blood pressure and vasodilator response during mental stress in obese children. Arq Bras Cardiol. 2011;96(6):490-7.

13. Gabbay FH, Krantz DS, Kop WJ. Triggers of myocardial ischemia during daily life in patients with coronary artery disease: physical and mental activities, anger and smoking. J Am Coll Cardiol. 1996;27:585-92.

14. Möller J, Theorell T, Faire U, Ahlbom A, Hallqvist J. Work related stressful life events and the risk of myocardial infarction. Case-control and case-crossover analyses within the Stockholm heart epidemiology programme (SHEEP). J Epidemiol Community Health. 2005;59:23-30.

15. Norton K, Olds T, editors. Antropometrica. Argentina: Biosystem; 2000.

16. Trombetta IC, Batalha LT, Rondon MUB, Laterza MC, Frazzatto E, Alves MJNN, et al. Gly16 + Glu27 beta2-adrenoceptor polymorphisms cause increased forearm blood flow responses to mental stress and handgrip in humans. J Appl Physiol (1985). 2005;98:787-94.

17. Stroop JR. Studies of interference in serial verbal reactions. J Exp Psychol Gen. 1992;121(1):15-23.

18. Kilic ID, Tanriverdi H, Fenkci S, Alkin F, Uslu S, Kaftan A. Noninvasive indicators of atherosclerosis in subclinical hypothyroidism. Indian J Endocrinol Metabolism. 2013;17(2):271-5.

19. Türemen EE, Çetinaslan B, Sarin T, Cantürk C, Tarkun I. Endothelial dysfunction and low grade chronic inflammation in subclinical hypothyroidism due to autoimmune thyroiditis. Endocri J. 2011;58(5):349-54.

20. Cabral MD, Teixeira PF, Silva NA, Morais FF, Soares DV, Salles E, et al. Normal flow-mediated vasodilatation of the brachial artery and carotid artery intima-media thickness in subclinical hypothyroidism. Braz J Med Biol Res. 2009;42(5):426-32.

21. Wilkinson IB, Webb DJ. Venous occlusion plethysmography in cardiovascular research: methodology and clinical applications. J Clin Pharmacol. 2001;52:631-46.

22. Lekakis J, Papamichael C, Anastasiou H, Alevizaki M, Desses N, Souvatzoglou A, et al. Flow-mediated, endothelium-dependent vasodilation is impaired in subjects with hypothyroidism, borderline hypothyroidism, and high-normal serum thyrotropin (TSH) values. Thyroid. 1997;7:411-14.

23. Taddei S, Caraccio N, Virdis A, Dardano A, Versari D, Ghiadoni $L$, et al. Low-grade systemic inflammation causes endothelial dysfunction in patients with Hashimoto's thyroiditis. J Clin Endocrinol Metab. 2006;91(12):5076-82. 
24. Ito A, Tsao PS, Adimoolan S, Kimoto M, Ogawa T, Cooke JP. Novel mechanism for endothelial dysfunction dysregulation of dimethylarginine dimethylaminohydrolase. Circulation. 1999;99:3092-5.

25. Lin KY, Ito A, Asagami T, Tsao PS, Adimoolam S, Kimoto M, et al. Impaired nitric oxide synthase pathway in diabetes mellitus role of asymmetric dimethylarginine and dimethylarginine dimethylaminohydrolase. Circulation. 2002;106:987-92.

26. Shanmugam N, Reddy MA, Guha M, Natarajan R. High glucoseinduced expression of proinflammatory cytokine and chemokine genes in monocytic cells. Diabetes. 2003;52:1256-64.

27. Dardano A, Ghiadoni L, Plantinga Y, Caraccio N, Bemi A, Duranti $E$, et al. Recombinant human thyrotropin reduces endothelium dependent vasodilation in patients monitored for differentiated thyroid carcinoma. J Clin Endocrinol Metab. 2006;91(10):4175-8.

28. Kamarck TW, Schwartz JE, Janicki DL, Shiffman S, Raynor DA. Correspondence between laboratory and ambulatory measures of cardiovascular reactivity: a multilevel modeling approach. Psychophysiol. 2003;40(5):675-83.

29. Ferreira MM, Teixeira Pde F, Mansur VA, Reuters VS, Almeida CP, Vaisman M. Monitorização ambulatorial da pressão arterial em pacientes normotensos com hipotireoidismo subclínico. Arq Bras Cardiol. 2010;94(6):806-12.

30. Toda N, Nakanishi-Toda M. How mental stress affects endothelial function. Pflugers Arch. 2011;462(6):779-94. 